\title{
Méthode d'extraction rapide des acides gras volatils libres des fromages
}

\author{
J.-L. BERDAGUE $(*)$ \\ I.N.R.A., Station Expérimentale Laitière - 39800 Poligny - France
}

\section{Résumé}

L'échantillon de fromage à analyser est homogénéisé mécaniquement après apport d'étalon interne dans une solution hydro-méthanolique à $10 \%$ d'eau (V/V) acidifiée par $1 \mathrm{ml}$ d'une solution aqueuse d'acide sulfurique à $6 \%(\mathrm{~V} / \mathrm{V})$.

Une brève centrifugation permet d'obtenir une phase liquide limpide contenant entre 50 et $70 \%$ des acides gras volatils libres de $\mathrm{C}_{2}$ à $\mathrm{C}_{6}$ présents dans le fromage. Cette phase est neutralisée par une solution de soude en présence de phénol-phtaléine. Les savons ainsi obtenus sont séchés par évaporation sous vide ou à pression ambiante. Les cristaux de savons sont rassemblés et pulvérisés pour être analysés par chromatographie en phase gazeuse.

La libération des $C_{4}$ et $C_{6}$ supplémentaires par méthanolyse des glycérides et l'estérification d'acides gras volatils libres par formation de méthyl-esters sont minimisés par apport d'eau dans le mélange d'extraction.

Cette technique permet de faire une extraction rapide des acides gras volatils du fromage de $\mathrm{C}_{2}$ à $\mathrm{C}_{6}$ compris, en vue de leur dosage par chromatographie en phase gazeuse.

Mots clés : Fromage - Méthode d'extraction - Acides gras volatils $\left(\mathrm{C}_{2}-\mathrm{C}_{6}\right)-$ Chromatographie phase gazeuse - Analyses.

\section{Summary}

A rapid extraction procedure of volatile free fatty acids in cheese

The cheese sample is mechanically homogeneized, after addition of an internal standard, with a $10 \%(\mathrm{~V} / \mathrm{V})$ aqueous methanolic solution acidified with $1 \mathrm{ml}$ of a $6 \%(\mathrm{~V} / \mathrm{V})$ aqueous sulphuric acid solution.

After centrifugation $5 \mathrm{~min}$ at $1500 \mathrm{~g}$, a limpid liquid phase is obtained containing between 50 and $70 \%$ of the $\mathrm{C}_{2}$ to $\mathrm{C}_{6}$ volatile free fatty acids present in the cheese sample. This phase is then neutralized by a $1 \% \mathrm{NaOH}$ solution containing phenolphtalein as indicator. The soaps obtained are dried by vacuum or at atmosphere

(*) Boursier CIFRE, Institut Technique du Gruyère. 
pressure evaporation. After pulverization, the soaps can be analyzed by gas-chromatography.

The release of additional $\mathrm{C}_{4}$ and $\mathrm{C}_{6}$ arising from hydrolysis of the glycerides by the methanol or conversly esterification of volatile free fatty acids giving methyl-esters are greatly reduced by the addition of water into the mixture of extraction.

This method provides a rapid and simple way of extracting volatile free fatty acids in cheese from $\mathrm{C}_{2}$ to $\mathrm{C}_{6}$ for gas chromatography analysis.

Key words : Cheese - Method of extraction - Volatile free fatty acid $\left(\mathrm{C}_{2}-\mathrm{C}_{6}\right)$ - Gas chromatography - Analysis.

\section{Introduction}

Plusieurs familles de méthodes d'extraction des acides gras volatils (A.G.V.) libres des fromages en vue de leur dosage par chromatographie en phase gazeuse existent actuellement, exploitant les propriétés physiques (caractère volatil) ou chimiques (ionisation du groupement carboxyle, solubilité) des A.G.V.

L'extraction par entraînement à la vapeur d'eau (RITTER et HANNI, 1960) suivie d'une neutralisation du distillat assure une bonne récupération des A.G.V. de $\mathrm{C}_{2}$ à $\mathrm{C}_{8}$ (VAmos-VigYazo et KISS-KuTZ, 1973) mais engendre des pertes en $\mathrm{C}_{2}$ comparativement à certaines méthodes d'extraction par solvant (KuZDZAL-SAvoie et KuzDzaL, 1967). L'entraînement à la vapeur a été utilisé pour doser les A.G.V. libres dans les fromages (Kuzdzal-Savoie et Kuzdzal, 1971, 1974).

Une méthode récente (FAVRE-BONVIN et ARPIN, 1984) permet l'injection directe du fromage à analyser dans un four balayé par un courant d'azote. Les composés volatils sont adsorbés sur support physique puis thermo-désorbés juste avant leur passage dans la colonne de chromatographie.

La séparation sur colonne d'acide silicique (HARPER, 1953; GrAY, 1975 ; Woo et LINDSAY, 1980 et 1982) ou sur résine échangeuse d'ions (NEEDs et al., 1983) offre de bons recouvrements. Cette technique est utilisée pour séparer les acides gras à longue chaîne (Woo et LindsAY, 1980 et 1982).

L'extraction par solvants offre de nombreuses possibilités. Dans le cas d'un solvant ou mélange de solvants non polaires (éther de pétrole, hexane par exemple), on peut entraîner la majeure partie des lipides du fromage, dont les A.G.V. Comme ces derniers, à l'inverse de la quasi-totalité des graisses, sont solubles en phase polaire (hydro-alcoolique par exemple), leur séparation se fait par partage entre la phase non polaire et la phase polaire. Cette séparation sera totale si l'on déplace les coefficients de partage en faveur de la phase polaire par apport de soude dans celle-ci (AGNet, 1979; KuzDzal-Savoie et al., 1967).

Diverses méthodes basées sur les phénomènes de partage entre plusieurs solvants ont été appliquées aux produits laitiers : beurre, matières grasses (GRAY, 1975 ; Kuzdzal-SAvoie et al., 1971) ou fromages (Kuzdzal-Savoie et al., 1967 ; AGNET, 1979) et permettent l'étude des A.G.V. libres y compris l'acide caproïque. L'extraction de l'acide acétique $\left(\mathrm{C}_{2}\right)$, propionique $\left(\mathrm{C}_{3}\right)$, butyrique $\left(\mathrm{C}_{4}\right)$ peut s'effectuer à partir d'une phase polaire aqueuse (FAMME et KNUDSEN, 1984 ; KEEN et WALKER, 1974). 
Le but de cette étude est de mettre au point une méthode rapide qui soit facilement utilisable dans le cadre des contrôles de routine par les laboratoires d'entreprise. La technique consiste à extraire les A.G.V. libres du fromage par une solution hydro-méthanolique. Le méthanol a été choisi comme solvant en raison de la bonne solubilité des A.G.V. de $\mathrm{C}_{2}$ à $\mathrm{C}_{6}$ dans le méthanol (WeAst, 1972), et de sa polarité qui confère à la solution des propriétés de solubilité vis-à-vis des lipides, intermédiaires entre celles de l'eau et celles de l'éthanol (TIAN, 1946), permettant ainsi une séparation directe et rapide des A.G.V. libres de l'ensemble de la matière grasse du fromage. Toutefois, pour limiter la réactivité du méthanol envers les glycérides, une faible quantité d'eau est ajoutée au solvant. Après homogénéisation du fromage dans la solution hydro-méthanolique, une centrifugation assure une bonne séparation entre la solution méthanolique et le culot contenant les protéines et les graisses, car le méthanol ne crée pas d'émulsion stable avec le fromage.

\section{Matériels et méthodes}

\section{A. Choix, préparation des fromages}

Les essais de mise au point de la méthode sont conduits sur des gruyères de Comté catégorie A (Comité Interprofessionnel du Gruyère de Comté) âgés de 4 mois. Ensuite, différents types de fromages sont analysés pour juger de l'efficacité de la technique d'extraction.

Pour chaque échantillon, une quantité suffisante de fromage de Comté (croûte éliminée) est râpée, mélangée puis tassée et congelée dans plusieurs récipents clos en attendant d'être analysée.

\section{B. Réactifs employés}

Méthanol, éther de pétrole (35-60), acide trichloracétique, acide sulfurique, hydroxyde de sodium (Normapur, Prolabo) et acide valérique (Sigma) sont de qualité analytique.

\section{Technique d'extraction des A.G.V. libres}

- Pesée de $5 \mathrm{~g}$ de fromage dans un tube à centrifuger en verre de $80 \mathrm{ml}$.

- Effectuer rapidement les opérations suivantes : $(\mathrm{V} / \mathrm{V})$;

- addition de $50 \mathrm{ml}$ d'une solution hydro-méthanolique à $10 \%$ d'eau

- addition de $1 \mathrm{ml}$ exactement mesuré de la solution d'étalon interne d'acide valérique $\left(\mathrm{C}_{5}\right)$ à $2 \%(\mathrm{~m} / \mathrm{V})$ dans de l'éther de pétrole $(35-60)$; rique ;

- acidification par $1 \mathrm{ml}$ d'une solution aqueuse à $6 \%(\mathrm{~V} / \mathrm{V})$ d'acide sulfu-

- mixtion du mélange $30 \mathrm{~s}$ à l'Ultra-Turrax en volume clos dans le tube à centrifuger jusqu'à obtention d'une suspension homogène ; 
$A G V(m g / 100 g$ de fromage)

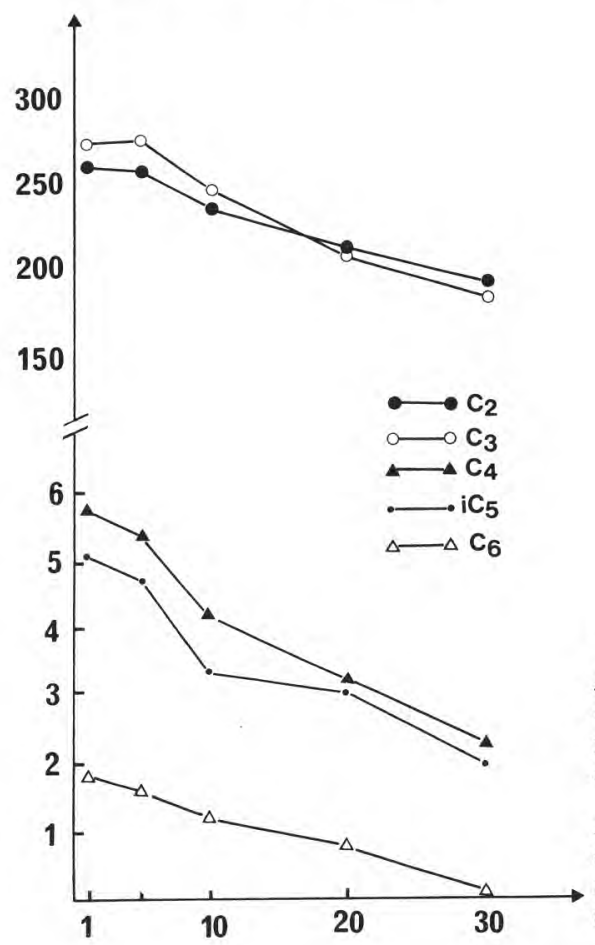

POIDS DE FROMAGE $(g)$
Fig. 1

Evolution des quantités d'acides gras volatils libres extraites ( $\mathrm{mg} / 100 \mathrm{~g}$ de fromage) en fonction $\mathrm{du}$ poids de fromage mis en ouvre pour un volume constant de solution hydro-méthanolique.

Evolution of the amounts of volatile free fatty acids (mg/100 $\mathrm{g}$ of cheese) measured according the amount of cheese used for a constant volume of aqueous methanol solution.

- après 5 min de centrifugation à $1500 \mathrm{~g}$, séparation du surnageant liquide puis neutralisation immédiate par de l'hydroxyde de sodium à $1 \%(\mathrm{~m} / \mathrm{V})$ en présence de phénol-phtaléine à $1 \%(\mathrm{~m} / \mathrm{V})$;

- arrêt de la neutralisation dès virage : apparition d'une légère nuance rose.

- Séchage sous vide ou à pression atmosphérique $\left(60-80^{\circ} \mathrm{C}\right)$.

- Pulvérisation du dépôt contenant les savons.

\section{Reprise des savons}

- $100 \mathrm{mg}$ de poudre bien homogénéisée sont acidifiés en tube fermé de $5 \mathrm{ml}$ avec $1 \mathrm{ml}$ d'une solution à $0,15 \mathrm{~g} / \mathrm{ml}$ d'acide trichloracétique dans de l'éthanol pur. Cette solution sera utilisée immédiatement.

- La dissolution de la poudre est obtenue après agitation (Vortex, position 2). La fraction insoluble contenant du sulfate de sodium donne un aspect trouble au liquide. 
- Laisser sédimenter le sulfate de sodium au fond du tube et n'injecter que le liquide clarifié.

- Pour éviter la formation de trichloracétate d'éthyle, cette sédimentation a lieu à basse température (environ $10 \mathrm{~min}$ dans le compartiment froid d'un réfrigérateur).

- La phase liquide est prête à être injectée dans le chromatographe.

\section{E. Chromatographie en phase gazeuse}

- Les A.G.V. sont séparés dans une colonne métal (Inox-GC, Supelco) longueur $1 \mathrm{~m}$, diamètre interne $2,5 \mathrm{~mm}$. Celle-ci est remplie d'un support (Chromosorb G/AW/DMCS, 100-120 mesh, Chrompack) imprégné à $10 \%$ en poids d'une phase polaire (FFAP : C20M + acide 2-nitrotéréphtalique ; CocHRANE, 1975). suit :

- Le matériel de chromatographie (Intersmat $121 \mathrm{DFL}$ ) est réglé comme

- température : du four $140^{\circ} \mathrm{C}$, de l'injecteur $200^{\circ} \mathrm{C}$, du détecteur $210^{\circ} \mathrm{C}$;

- débits : gaz vecteur azote $30 \mathrm{ml} \cdot \mathrm{min}^{-1}$, hydrogène $30 \mathrm{ml} \cdot \mathrm{min}^{-1}$, azoteoxygène (50-50) $300 \mathrm{ml} \cdot \mathrm{min}^{-1}$.

- Le volume d'injection est de $1,5 \mu 1$.

\section{F. Analyses de fromages non affinés}

Dans le cas de fromages très jeunes (âgés de quelques jours), de fortes quantités d'acide lactique peuvent être présentes. Comme le temps de rétention de l'acide lactique est long ( 3 min environ) un pic très aplati peut alors interférer avec les pics des A.G.V. dans le cas d'analyses en série comportant des injections répétées. Ce problème disparaît en analysant des fromages affinés ayant peu d'acide lactique.

\section{Résultats et discussion}

\section{A. Mise au point de la méthode}

\section{Détermination du rapport optimum fromage/solution hydro-méthanolique}

Différentes quantités de Gruyère de Comté, allant de 1 à $30 \mathrm{~g}$ et préparées selon I.A. sont extraites par un volume constant de $50 \mathrm{ml}$ de solution hydrométhanolique à $10 \%(\mathrm{~V} / \mathrm{V})$. On constate que, pour des prises d'essai comprises entre 1 et $10 \mathrm{~g}$ de fromage, la centrifugation du broyat permet d'obtenir une phase méthanolique limpide. A partir de $20 \mathrm{~g}$, on observe un trouble et un surnageant jaune de graisses du fromage. Les concentrations maximum d'A.G.V. sont obtenues pour des poids de fromage extraits de 1 à $5 \mathrm{~g}$. Dans la suite des essais, la prise de $5 \mathrm{~g}$ de fromage sera retenue (rapport fromage/solution méthanolique $\mathrm{V} / \mathrm{V}$ de 0,1 ) car elle donne suffisamment de savons pour l'analyse. 


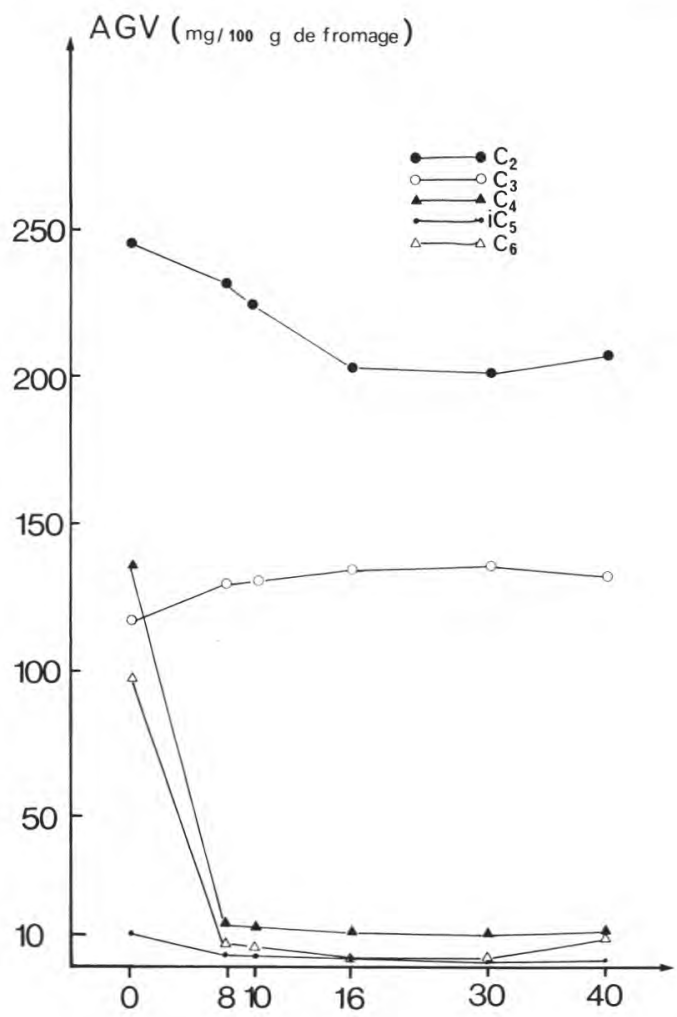

Fig. 2

Quantités d'acides gras volatils libres obtenues en une extraction en fonction de la proportion d'eau dans le mélange d'extraction eau-méthanol.

Amount of volatile free fatty acids obtained in a single extraction according to the proportion of water into the "watermethanol " extraction mixture.

PROPORTION D'EAU DANS LE MELANGE DÉETRACTION (\%)

\section{Influence du rapport eau/méthanol dans la phase hydro-méthanolique} d'extraction

En utilisant un volume de solution hydro-méthanolique constant de $50 \mathrm{ml}$, acidifé par $1 \mathrm{ml}$ d'une solution aqueuse à $6 \%(\mathrm{~V} / \mathrm{V})$ d'acide sulfurique, dont la proportion d'eau varie de 0 à $30 \%$, deux types de phénomènes sont mis en évidence : $30 \%$;

- une mauvaise séparation des phases dès que la proportion d'eau atteint

- une méthanolyse des glycérides en absence d'eau; la figure 2 montre qu'en dessous de $8 \%$ d'eau dans le méthanol il y a apparition de $\mathrm{C}_{4}$ et $\mathrm{C}_{6}$ supplémentaires provenant des glycérides.

Un simple apport d'eau agit de manière importante sur les cinétiques et équilibres des réactions de la liaison ester (hydrolyse et estérification) (ARNAUD, 1973 ; BAMFORD et TePPER, 1972). Cet apport d'eau est d'autant plus essentiel que les courtes chaînes des glycérides sont les plus sensibles à la méthanolyse (BAMFORD et TEPPER, 1972). 
La proportion de $10 \%$ d'eau dans le méthanol a été retenue car elle apparait être un bon compromis entre un excellent partage (après centrifugation) et le risque possible de méthanolyse des glycérides.

Afin de vérifier, dans des conditions chimiques plus sévères, le rôle de l'apport d'eau sur la méthanolyse des glycérides, deux séries de trois extractions successives (où le culot de fromage est soumis à une nouvelle extraction après chaque centrifugation) ont été effectuées sur deux échantillons identiques de fromage de Comté. Le méthanol pur a été utilisé dans la première série, la solution méthanolique à $10 \%$ d'eau dans la seconde. La figure 3 confirme de façon très nette qu'en absence d'eau il y a une augmentation importante des quantités de $\mathrm{C}_{4}$ et $\mathrm{C}_{6}$ extraites.

\section{Influence de l'acidification du milieu d'extraction}

L'acidification a pour but de libérer les A.G.V. présents sous forme de sels éventuellement peu ou non solubles en milieu hydro-méthanolique. L'apport de $1 \mathrm{ml}$ d'acide sulfurique à $6 \%$ permet d'abaisser le $\mathrm{pH}$ du milieu à une valeur inférieure au $\mathrm{pK}$ des acides gras volatils. Le regroupement de dix extractions menées avec ou sans acidification sur des fromages de Comté montre que l'on extrait davantage de $\mathrm{C}_{2}$ et $\mathrm{C}_{3}$ en milieu acide à $\mathrm{pH} 4$ (tabl. 1) car on libère plus d'A.G.V. de leurs sels.

Afin d'estimer le risque d'hydrolyse acide, des graisses de fromage (dont les acides gras libres ont été préalablement éliminés par lavage en ampoule à décanter en présence d'une phase hexane et d'une phase aqueuse à $5 \%$ de carbonate de potassium) ont été émulsionnées dans la solution hydrométhanolique acidifiée. La chromatographie sur couche mince de la fraction méthanolique selon la technique de Mangold et KammerecK (1962) n'a pas présenté de taches d'acides gras libres consécutives à hydrolyse des glycérides.

\section{B. Répétabilité}

Un échantillon de Comté a été analysé dix fois selon la technique décrite. Chaque analyse correspond à la moyenne de deux déterminations par chromatographie en phase gazeuse. Les résultats du tableau 2 montrent que le coefficient de variation de répétabilité est en relation inverse avec la quantité d'acides gras volatils mesurée. La mauvaise répétabilité de la mesure d'iC $\mathrm{C}_{5}$ peut s'expliquer par le fait qu'il apparaît un petit pic non identifié entre $\mathrm{iC}_{5}$ et $\mathrm{C}_{5}$ dont la surface mal définie par l'intégrateur est parfois reportée sur iCs.

\section{Caractéristiques d'extraction des A.G.V. libres}

\section{Rendement d'extraction}

La capacité de la méthode à extraire le maximum d'A.G.V. libres dans le fromage a été testée en effectuant trois extractions consécutives (avec reprise du culot de centrifugation) sur un même échantillon de fromage. Les savons obtenus par les extractions 1,2 et 3 ont permis l'obtention de chromatogrammes qui visualisent l'effet des extractions successives (fig. 4). 

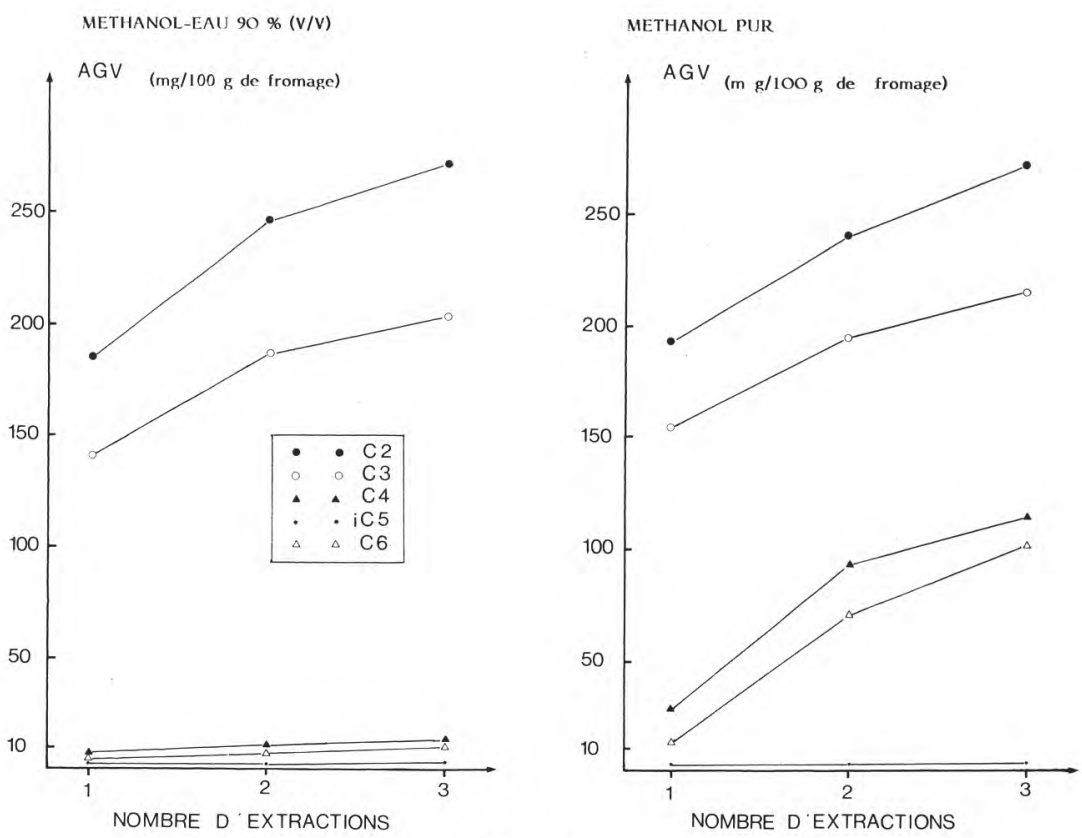

Fig. 3

Evolution des quantités d'acides gras volatils (en $\mathrm{mg} / 100 \mathrm{~g}$ de fromage) extraites en fonction du nombre d'extractions et de la phase utilisée.

Evolution of the amounts of volatile free fatty acids ( $\mathrm{mg} / 100 \mathrm{~g}$ of cheese) extracted according to the number of extractions and of the phase used.

\section{TABLEAU I}

Influence de l'acidification du milieu sur la quantité d'A.G.V. libres extraite ( $\mathrm{mg} / 100 \mathrm{~g}$ de fromage). Valeurs moyennes de 10 extractions comparées

Influence of acidification of the extraction mixture on the amount of volatile free fatty acids ( $\mathrm{mg} / 100 \mathrm{~g}$ of cheese). Mean values of ten comparative extractions

\begin{tabular}{l|c|c}
\hline & Acidification $(\mathrm{pH} \# 4)$ & Sans acidification $(\mathrm{pH} \#$ 6,9) \\
\hline $\mathrm{C}_{2}$ & 205 & 180 \\
$\mathrm{C}_{3}$ & 152 & 137 \\
$\mathrm{C}_{4}$ & 5,4 & 5,2 \\
$\mathrm{C}_{5}$ & 1,8 & 1,9 \\
$\mathrm{C}_{6}$ & 3,7 & 3,5 \\
\hline
\end{tabular}


TABLEAU II

Répétabilité de la méthode d'extraction (1 échantillon, 10 répétitions)

Repetability of extraction method (1 sample, 10 replicates)

\begin{tabular}{c|c|c|c}
\hline & $\begin{array}{c}\mathrm{x} \\
(\mathrm{mg} / 100 \mathrm{~g} \\
\text { de fromage }\end{array}$ & or & $\mathrm{CV} \%$ \\
\cline { 2 - 3 } $\mathrm{C}_{2}$ & 242 & 22 & 9,3 \\
$\mathrm{C}_{3}$ & 3.17 & 12 & 3,8 \\
$\mathrm{C}_{4}$ & 6,9 & 0,6 & 8,7 \\
$\mathrm{iC}_{5}$ & 5,2 & 1,7 & 32,7 \\
$\mathrm{C}_{6}$ & 4,8 & 0,9 & 18,8 \\
\hline
\end{tabular}

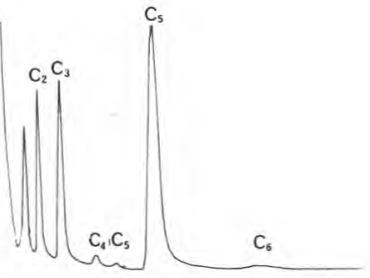

EXTRACTION

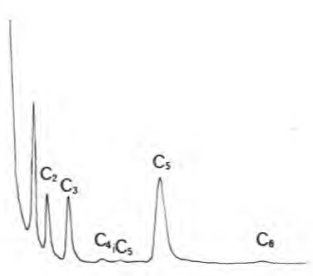

EXTRACTION 2

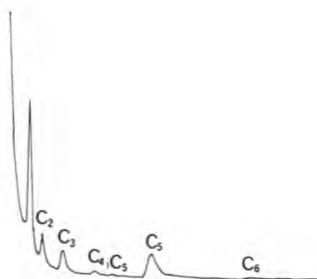

EXTRACTION 3

Fig. 4

Chromatogrammes obtenus au cours de trois extractions successives.

Chromatograms obtained after three successive extractions.

Afin de préciser les rendements après la première, deuxième et troisième extraction, les quantités des différents A.G.V. sont calculées par rapport à la surface totale d'étalon interne mesurée après la troisième extraction. Pour rendre plus explicites les comparaisons entre chaque acide gras volatil, les rendements d'extraction ont été calculés en admettant $100 \%$ de rendement à la troisième extraction (tabl. 3).

Les rendements d'extraction des différents acides gras volatils, dont dépend la justesse de la méthode, sont variables selon les A.G.V. En une extraction, ils sont compris entre 60 et $69 \%$ pour $\mathrm{C}_{2}, \mathrm{C}_{3}$, iC $\mathrm{C}_{5}$ et entre 47 et $49 \%$ pour $\mathrm{C}_{4}$ et $\mathrm{C}_{6}$.

Les rendements exprimés ici sont la résultante de phénomènes complexes qui sont fonction de la nature et de la solubilité des phases, de la taille, de la forme et de la composition des particules solides, du temps de contact entre phases, de l'agitation et de la température du milieu. Différentes variétés de fromages peuvent avoir des rendements d'extraction que seule l'expérience permet 
TABLEAU III

Rendement d'extraction des acides volatils libres (\%) après 1, 2 et 3 extractions Efficiency of volatile free fatty acids extraction (\%) after 1, 2 and 3 extractions

\begin{tabular}{c|c|c|c}
\hline & $\mathrm{E}_{1}$ & $\mathrm{E}_{1}+\mathrm{E}_{2}$ & $\mathrm{E}_{1}+\mathrm{E}_{2}+\mathrm{E}_{3}$ \\
\hline $\mathrm{C}_{2}$ & 68 & 91 & 100 \\
$\mathrm{C}_{3}$ & 69 & 92 & 100 \\
$\mathrm{C}_{4}$ & 49 & 77 & 100 \\
$\mathrm{iC}_{5}$ & 60 & 76 & 100 \\
$\mathrm{C}_{6}$ & 47 & 69 & 100 \\
\hline
\end{tabular}

de mesurer. Comme il ne s'agit pas d'une technique d'extraction par épuisement, la concentration et la composition des A.G.V. dans le fromage risquent d'influencer les caractéristiques de l'extraction. On constate d'après le tableau 3 que l'extraction des $\mathrm{C}_{4}$ et $\mathrm{C}_{6}$ est plus difficile dans le fromage de Comté analysé.

2. Estimation de la capacité d'extraction de la méthode par mesure du taux de récupération d'ajouts dosés

Trois extractions consécutives ont été effectuées sur un échantillon de fromage de Comté prélevé $20 \mathrm{~h}$ après la fabrication auquel ont été incorporées les quantités d'A.G.V. suivantes :

$-\mathrm{C}_{2}, \mathrm{C}_{3}=300 \mathrm{mg} / 100 \mathrm{~g}$ de fromage ;

- $\mathrm{C}_{4}, \mathrm{C}_{6}=50 \mathrm{mg} / 100 \mathrm{~g}$ de fromage ;

$-\mathrm{iC}_{5}=20 \mathrm{mg} / 100 \mathrm{~g}$ de fromage.

Dans le calcul des taux de récupération, il est tenu compte des quantités d'A.G.V. déjà présentes dans le fromage.

Par comparaison avec l'essai de détermination des rendements d'extraction, il semble que les $\mathrm{C}_{4}$ et $\mathrm{C}_{6}$ soient plus facilement extraits lors de la première extraction quand ils sont ajoutés au fromage (tabl. 4).

Le risque d'une perte d'acides gras volatils consécutif à une formation de méthyl-esters est minime pour deux raisons. D'une part, les conditions d'extraction (présence d'eau, acidification du milieu, température ambiante et temps de contact) placent cette réaction dans des conditions défavorables, d'autre part, après neutralisation, les A.G.V. libres ne peuvent plus être estérifiés (BANNON, 1982).

D. Comparaison avec une méthode d'extraction par un mélange d'oxyde d'éthyle et d'éther de pétrole (35-60)

La méthode prise en référence est l'adaptation par AgNET (1979) d'une technique déjà décrite (KuZDZAL-SAvoie, 1966), permettant le dosage des A.G.V. 


\section{TABLEAU IV}

Recouvrement (\%) des acides gras volatils libres incorporés au fromage après 1, 2 et 3 extractions

Percent recovery of volatile free fatty acids incorporated in cheese after 1,2 and 3 extractions

\begin{tabular}{c|c|c|c}
\hline & $\mathrm{E}_{1}$ & $\mathrm{E}_{1}+\mathrm{E}_{2}$ & $\mathrm{E}_{1}+\mathrm{E}_{2}+\mathrm{E}_{3}$ \\
\hline $\mathrm{C}_{2}$ & 67 & 84 & 89 \\
$\mathrm{C}_{3}$ & 68 & 86 & 93 \\
$\mathrm{C}_{4}$ & 62 & 82 & 87 \\
$\mathrm{iC}_{5}$ & 62 & 79 & 89 \\
$\mathrm{C}_{6}$ & 60 & 83 & 85 \\
\hline
\end{tabular}

libres dans les fromages. Il s'agit d'une extraction des graisses et des A.G.V. libres par épuisement sous flux d'un mélange 50-50 (V/V) d'oxyde d'éthyle et d'éther de pétrole (35-60).

Les A.G.V. sont séparés des graisses par partage entre la phase éthérée ou apolaire et une phase hydro-éthanolique après neutralisation par une solution d'hydroxyde de sodium.

Vingt-cinq Comtés d'âge et d'origine différents ont été analysés par les deux méthodes. Comme aucun de ces fromages n'avait une teneur élevée en $\mathrm{C}_{4}$, une série supplémentaire de 20 échantillons de fromages butyriques a été analysée par les deux méthodes (Courroye, 1984), afin de mieux définir la précision de la technique pour l'extraction du $\mathrm{C}_{4}$. Les résultats sont regroupés sur la figure 5 . On constate pour chaque acide gras une bonne corrélation entre les deux méthodes $(0,83<\mathrm{r}<0,99)$. Les plages de concentration pour lesquelles a été testée la méthode sont variables selon les A.G.V. : $\mathrm{C}_{2}$ et $\mathrm{C}_{3}=0$ à $300 \mathrm{mg}$ par $100 \mathrm{~g}$ de fromage, $\mathrm{C}_{4}=0$ à $200 \mathrm{mg}$ par $100 \mathrm{~g}$ de fromage, $\mathrm{iC}_{5}$ et $\mathrm{C}_{6}=0$ à 6 et 0 à $20 \mathrm{mg}$ par $100 \mathrm{~g}$ de fromage. Les coefficients de variation (C.V.) des différents A.G.V. seront donc à considérer dans les plages de variation précitées. Il apparaît que l'extraction de $C_{4}$ et $C_{6}$ par le méthanol manque de sensibilité jusqu'à $30 \mathrm{mg}$ par $100 \mathrm{~g}$ de fromage pour $\mathrm{C}_{4}$ au moins jusqu'à $20 \mathrm{mg}$ par $100 \mathrm{~g}$ de fromage pour $\mathrm{C}_{6}$. Ce manque de sensibilité peut provenir du fait qu'une phase apolaire (éther de pétrole) solubilise la phase lipidique (globules gras, cristaux mixtes des triglycérides) contrairement au mélange éthanol-eau, qui assure donc une moindre extraction des acides gras volatils. Au-delà de $30 \mathrm{mg}$ par $100 \mathrm{~g}$ de fromage, la mesure de $\mathrm{C}_{4}$ apparaît bonne. Ne disposant d'aucun fromage très riche en $\mathrm{C}_{6}$, il n'a pas été possible de tester l'efficacité de la méthode au-delà de $20 \mathrm{mg}$ par $100 \mathrm{~g}$ de fromage. 

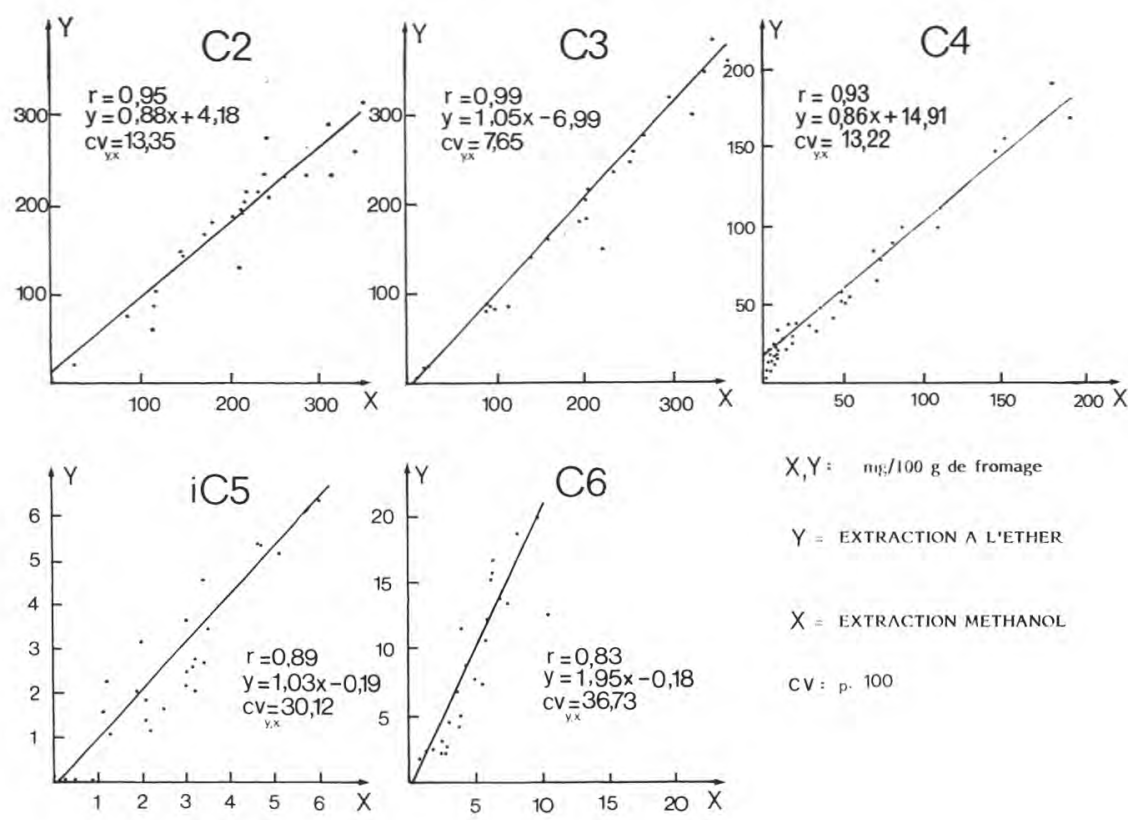

Fig. 5

Comparaison des résultats de chromatographie en phase gazeuse des acides gras volatils obtenus à partir d'échantillons de fromage de Comté après extraction par un mélange eau-méthanol $(y)$ et un mélange d'oxyde d'éthyle et d'éther de pétrole.

Comparison of gas chromatographic results of volatile free fatty acids obtained on Comté cheese samples after extraction by the water-methanol mixture $(y)$ and by the diethylether and petroleum spirit mixture.

\section{E. Application de la technique à divers types de fromages}

Le tableau 4 présente des résultats plausibles compte tenu de ceux déjà obtenus dans la littérature (KuzDzAL-SAvoIE, 1966-1974). Les valeurs les plus élevées en $\mathrm{C}_{2}$ et $\mathrm{C}_{3}$ correspondent à des pâtes cuites pressées, $\mathrm{iC}_{4}$ et $\mathrm{iC}_{5}$ apparaissent relativement abondants dans les pâtes persillées et dans un fromage d'Epoisses très fait. Les quantités de $\mathrm{C}_{4}$ dans des Comtés à ouverture butyriques sont supérieures à celles observées dans des Comtés de qualité A. Des quantités relativement fortes de $\mathrm{C}_{4}$ ont été mesurées sur un fromage d'Epoisses.

\section{Conclusion}

En utilisant la méthode d'extraction proposée, il a été montré que le risque de doser des $\mathrm{C}_{4}$ et $\mathrm{C}_{6}$ supplémentaires issus de la méthanolyse des glycérides et 


\section{TABLEAU V}

Quantités ( $m g / 100 \mathrm{~g}$ de fromage) d'acides gras volatils libres trouvés dans quelques variétés de fromages analysées

Amounts of volatile free fatty acids ( $\mathrm{mg} / 100 \mathrm{~g}$ of cheese) found in some cheese varieties

\begin{tabular}{lrrrrrrr}
\hline A.G.V. en mg/100 g de fromage & $\mathrm{C}_{2}$ & $\mathrm{C}_{3}$ & $\mathrm{iC}_{4}$ & $\mathrm{C}_{4}$ & $\mathrm{iC}_{5}$ & $\mathrm{iC}_{6}$ & $\mathrm{C}_{6}$ \\
Comté (4 mois) & 202 & 135 & - & 11 & 2 & - & 3 \\
& 265 & 200 & - & 10 & 3 & - & 6 \\
Comté (1 mois 1/2) & 104 & 27 & - & 8 & 7 & - & 2 \\
Comté (20 h) & 25 & 10 & - & 3 & - & - & 3 \\
Comté butyrique (4 mois) & 151 & 90 & - & 54 & - & - & 3 \\
& 125 & 100 & - & 45 & - & - & 2 \\
& 84 & 40 & - & 224 & 4 & - & 1 \\
Emmental propio butyrique (4 mois) & 173 & 171 & - & 33 & 3,4 & - & 1 \\
Reblochons fermiers & 40 & 12 & - & 12 & 4 & - & 10 \\
(moyenne sur 15 fromageries) & 38 & 4 & - & 4 & 1 & - & 1 \\
Reblochons laitiers & & & & & & & \\
(moyenne sur 5 fromageries) & 82 & 57 & 3 & 27 & 26 & - & 8 \\
Gorgonzola (peu affiné) & 29 & 9 & 1 & 12 & 17 & - & 9 \\
Bleu d'Auvergne (peu affiné) & 29 & 5 & 4 & 17 & 40 & 5 & 6 \\
Bleu d'Auvergne & 30 & - & - & 4 & 10 & - & 0 \\
Camembert (plâtreux) & 176 & 149 & 32 & 234 & 43 & 12 & 16 \\
Epoisses (très affiné) & & & & & & & \\
\hline
\end{tabular}

celui de ne pas doser des acides gras volatils par formation d'esters méthyliques sont très réduits par un apport d'eau dans le mélange d'extraction.

Cette technique permet une bonne appréciation de la composition des A.G.V. libres de $\mathrm{C}_{2}$ à $\mathrm{C}_{6}$ dans les fromages par comparaison avec la méthode prise en référence. Une sous-estimation des teneurs en $\mathrm{C}_{4}$ et $\mathrm{C}_{6}$ apparaît néanmoins lorsque les concentrations sont inférieures respectivement à 30 ou $20 \mathrm{mg}$ par $100 \mathrm{~g}$ de fromage.

La méthode permet de faire une mesure précise de la teneur en $C_{4}$ pour des fromages butyriques ayant plus de $30 \mathrm{mg}$ par $100 \mathrm{~g}$ de fromage de cet acide.

L'avantage de cette technique réside dans la simplicité et la rapidité d'extraction des acides gras volatils du fromage.

Lors des manipulations, il est recommandé de prendre les précautions d'usage afin d'éviter les dangers concernant l'emploi fréquent du méthanol.

Reçu le 17-4-1985.

Accepté pour publication le 20-11-1985

\section{Références bibliographiques}

AgNET Y., 1979. Note technique non publiée sur l'analyse des acides gras volatils de C2 à C6. Laboratoire Départemental d'Analyses Agricoles - 39800 Poligny. 
ARnaud P., 1973. Cours de chimie organique. Gauthier-Villars, Paris, 203-204.

BAMFoRD C.H., TePPER C.F.H., 1972. Comprehensive chemical ester formation and hydrolyse and related creations. Elsevier, Amsterdam, 10, 131, 142.

Bannon C.D., Breen G.J., Craske J.D., 1982. Analysis of fatty acid methyl esters with high accuracy and reliability. J. Chromatogr., 247, 71-89.

COMité INTERPRofessionnel du GRUYÈre de COMTÉ (C.I.G.C.), rue des Ecoles - 39800 Poligny. Grille de classement technique du fromage de Comté.

Cochrane C.G., 1975. A review of the analysis. J. Chromatogr. Sci., 13, 440-447.

COURROYE M., 1984. Communication personnelle. Institut Technique du Gruyère, Rioz - 70190.

FAMME P., KNUDSEN J., 1984. Direct gas chromatographic determination of short chain $\left(\mathrm{C}_{2}-\mathrm{C}_{4}\right)$ volatile fatty acids in aqueous solutions. Comp. Biochem. Physiol., 77, 617-618.

Favre-Bonvin J., ARPIN N., 1984. Une nouvelle méthode de dosage des A.G.V. Rev. Lait. Fr., 434, 38-40.

GRAY I.K., 1975. A rapid quantitative method for the separation of free fatty acids from other lipids. N.Z.J. Dairy Sci. Technol., 10, 158-162.

HARPER W.J., 1953. Direct chromatographic determination of acetic, propionic and butyric acids in cheese. I. Dairy Sci., 36, 808-816.

KEEN A.R., WALKER N.J., 1974. The determination of acetic, propionic and butyric acids in cheese. J. Dairy Res., 41, 397-404.

KuzDzaL-SavoIe S., KuZDZaL W., 1966. Les acides gras libres du fromage, Lait, 461-462, 9-23.

Kuzdzal-Savore S., Kuzdzal W., Langlors D., 1971. Note technique. Dosage des acides gras libres. Lait, 508, 534-544.

Kuzdzal-Savoie S., KuzdzaL W., 1974. L'analyse par chromatographie en phase gazeuse des acides gras volatils libres des fromages et l'appréciation organoleptique. Spectra 2000, 9, 62-66.

Kuzdzal-Savoie S., Kuzdzal W., 1971. Dosage des acides gras libres volatils dans quelques types de fromages français. Tech. Lait., 717, 11-14.

Mangold H.K., Kammereck R., 1962. J. Am. Oil Chem. Soc., 39, 201.

NEEDS E.C., Ford G.D., OWEN A.J., 1983. A method for the quantitative determination of individual free fatty acids in milk by ion exchange resin adsorption and gas liquid chromatography. J. Dairy Res., 50, 321-329.

RITTER W., HANNI H., 1960. Die Anwendung der Gaschromatographie in der Milchwissenschaft. Milchwissenschaft, 15, 296.

Tian A., Roche J., 1946. Précis de chimie, Masson, Paris, 564.

Vamos-Vgyazo L., Kiss-Kutz N., 1974. Estimation of volatile acids in Emmental cheese by gas-liquid chromatogrphy. Acta Aliment., 3, 309-325.

WEAST R.C., 1971-1972. Handbook of chemistry and physics. Physical constants of organic compounds. The chemical rubber, Cleveland.

Woo A.H., LIDSAY R.C., 1980. Method for the routine quantitative gas chromatographic analysis of major free fatty acids in butter and cream. J. Dairy Sci., 63, 1058-1064.

Woo A.H., LINDSAY R.C., 1982. Rapid method for quantitative analysis of free fatty acids in Cheddar cheese. J. Dairy Sci., 65, 1102-1108. 